\title{
Examining Fourth Year Engineering Student Perceptions of Graduate ATtribute Competencies: Year Two
}

\author{
Jillian Seniuk Cicek, Paul Labossiere and Sandra Ingram \\ Faculty of Engineering, University of Manitoba, Winnipeg, MB, Canada R3T 5V6 \\ umseniuk@cc.umanitoba.ca
}

\begin{abstract}
As the Engineering Faculty at the University of Manitoba shifts its curriculum from an input-based to an outcomes-based pedagogy, data from diverse sources are being collected. Among them, indirect data are being gathered from students using a student exit survey. The survey has been developed over the past three years to explore graduating students' perceptions of their aptitudes and their engineering program's strengths and weaknesses in regards to the 12 CEAB graduate attributes. It is comprised of the 12 attributes, with each attribute further defined by six indicators. These indicators reflect the levels of Bloom's Taxonomy of Educational Objectives in the Cognitive Domain: knowledge, comprehension, application, analysis, synthesis and evaluation. The student exit survey was first administered to graduating mechanical engineering students at the end of Fall semester 2012 and then at the end of Fall semester 2013. This paper describes the second year of the study, and discusses the data comparatively with the findings from the first year. This study offers the Engineering Faculty an understanding of their Mechanical Engineering students' experiences with and perceptions of the CEAB graduate attributes. It will be used to provide feedback at instructor, program and faculty levels as the University of Manitoba's Faculty of Engineering continues to implement its cycle of program development and improvement.
\end{abstract}

Keywords: student exit survey; outcomes-based assessment; graduate attributes; Bloom's Taxonomy; accreditation

\section{INTRODUCTION}

As part of the requirements for earning CEAB accreditation, engineering faculties must have a comprehensive assessment protocol in place to assess their program outcomes, which among other requirements, necessitates gathering data from a number of sources. According to engineering education researchers, there are five necessary steps when preparing for accreditation evaluation [16][23]. The first is to: "Establish a protocol for collecting contributions/ feedback about your engineering program from different groups of stakeholders, such as students presently enrolled in the program, alumni, and industry" [23].

There are several methods available for gathering data from stakeholders [23], comprising of quantitative and qualitative assessments [17] from both direct and indirect sources. Direct sources include assignments, tests, exams and standardized tests [21][23], traditional methods of assessment that are part of most, if not all, engineering programs. Indirect sources of data can be collected using SEEQs, or student evaluations of teaching; alumni surveys, which ask alumni to rate their preparedness for the field; Industry surveys or questionnaires, which ask employers to rate engineering graduates' readiness for their profession [21][23]; and exit surveys, where students can rate both program objectives and graduate attribute outcomes and perceived importance, as well as their own perceived preparation for their discipline [17][[23].

Instruments such as surveys, questionnaires and interviews measure the perceptions of participants rather than the direct evidence of the learning outcomes themselves [15]. That is why they are considered indirect sources of data. These perceptions can color the way participants view their learning experience, and so too can a participant's personality: "individual characteristics of students make a difference in what they claim to end up gaining... Those whose primary motivational orientation is one of personal mastery tend to believe they take away more of the core engineering competency" [15]. As well, there is evidence that faculty and students have varied acuities when it comes to teaching, learning and assessing [8]. Despite these potential limitations, measuring perceptions is an important part of a faculty's assessment protocol, as it is another way to measure the success or potential shortcomings of a program from the point of view of different stakeholders. To provide more insight into curricular strengths and weaknesses for the purposes of assessing educational competencies in a program, it is a good idea to compare the perceptions of students with the perceptions of faculty and other stakeholders: "A careful examination of the perceptions of students about educational efforts for these outcomes and how these perceptions do or do not match those of faculty and administrators can provide an important foundation to the assessment of the success of curricular efforts" [8].

In the Faculty of Engineering at the University of Manitoba, several assessment protocols are already in 
place. In particular, for the Mechanical Engineering program, these protocols include a student exit survey that is given to students in their last semester before graduation. The student exit survey was first developed and implemented in the Biosystems program in the 2011 academic year to measure students' perceptions of how well their engineering program at the University of Manitoba prepared them in regard to the $12 \mathrm{CEAB}$ graduate attributes [20]. It has since been revised and adopted by the Mechanical Engineering program as well. Last year, the survey was changed to reflect Bloom's Taxonomy [2][20]. The survey was administered to fourth year students in the Mechanical Engineering program taking MECH 4860 Engineering Design. Findings were presented at the CEEA 2013 conference [20]. This year, the survey has been revised and administered again with the objective to continue to measure the perceptions of fourth year Mechanical Engineering students in regards to the graduate attributes in their own development and in the program's curriculum. This paper documents some of the revisions of this year's survey, and presents some of the findings in comparison to the data from the first year of the study.

\section{SURVEY DEVELOPMENT}

The second iteration of the student exit survey was developed using Bloom's Taxonomy of Educational Objectives in the Cognitive Domain as a framework to organize each of the $12 \mathrm{CEAB}$ graduate attributes into six indicators [2]. Each indicator reflected one of Bloom's six cognitive levels: knowledge, comprehension, application, analysis, synthesis and evaluation. Students were asked to rank their proficiency of the cognitive levels for each attribute on a three-point scale to indicate whether they i) did not develop the skill/ability; (ii) were introduced to the skill/ability but did not master it; or (iii) mastered the skill/ability within the Mechanical Engineering curriculum [20]. Additionally, students were asked to choose the three strongest and three weakest attributes in regards to the Mechanical engineering program and with respect to their own knowledge, skills and behaviors [20].

For the second year, it was determined that it was important to maintain the integrity of the survey in order to begin to build a longitudinal understanding of students' perceptions of the program and of their own aptitudes for the purpose of implementing a feedback loop [24]. However, based on the data from the first year's administration, it was necessary to make some changes to the structure and language of the survey. As stated above, when responding to the survey in year one, students were only given three choices to rate how attributes were delivered in the program and their resulting prowess. It was found that a number of students created two additional categories by choosing to mark on the line between the three options. Evidently, students desired a larger scale to express their perceptions. Therefore, the survey was revised to reflect a five-point scale.

The additional change to the survey was in the language used to describe students' competency in regards to the attributes. For example, in the first year students were asked if they did not develop, were introduced but did not master or mastered the skills/ability reflected by the indicator. A number of problems were identified by these language choices. Firstly, one category emphasized the role of the instructor by using the word introduced, indicating that some of the responsibility for student learning was the instructor's. On the other hand, the remaining two categories did not describe whether or not the attribute was introduced, and only the words develop and master were used, which excluded the role of the instructor and placed full responsibility for learning squarely onto the student. Therefore, the three categories were essentially asking for two different assessments, which could potentially skew the data. Secondly, the word master was never defined, again possibly leading to different interpretations and thereby a variety of assessments, perhaps distorting the results. Finally, it was determined that there was too great a divide between did not develop, did not master and master, with no middle ground to describe attribute aptitude. As a result of these findings, for the second installment of the survey, the phrase introduced to the skill/ability was used in the descriptor for each ranking, and the language in regards to students' abilities was changed to poor, adequate, good and excellent to provide students with additional, and arguably more familiar categories, as these words are representative of rubric descriptors, and are familiar to many students. The choices also reflect an increase in aptitude in smaller increments to more aptly describe attribute abilities. (The exact wording of the survey descriptors for each ranking can be found in Appendix A.)

\section{METHODS}

An ethics application was submitted to Education/Nursing Research Ethics Board (ENREB), the governing research ethics board for the Faculty of Engineering at the University of Manitoba. Once approved, the student exit survey was administered to graduating Mechanical Engineering students in December 2013. Distribution was as follows: The instructor of MECH 4860 Engineering Design handed team leaders a manila envelope of surveys with a recruitment letter stapled to the envelope. The team leaders were asked to distribute the surveys to their team members. Email requests were also sent to students in additional recruitment attempts. Completed surveys were either collected by team leaders and placed into an unmarked envelop and left in the Mechanical Engineering office, or emailed to the co-researcher. This was done to assure 
students' anonymity and prevent any conflict of interest vis-à-vis the principal researcher, who was also the course instructor of MECH 4860 Engineering Design. Thus, although the survey was not connected to the course, it was important to protect students' potential vulnerability in this regard. Quantitative descriptive statistics was used to numerically summarize the data.

\section{FINDINGS}

A major change between the first year and second year of the study was found in the response rate. In 2012-13, there were 76 responses out of 78 students, which is a $97 \%$ response rate. For this second year of the study, 2013-14, 23 students responded to the survey out of 81 students: a $28 \%$ response rate. This difference could possibly be explained by the timing of the distribution of the surveys. During the first year of the study, the exit survey was distributed to team leaders during the semester. Therefore, team leaders were easily able to encourage their team members to complete the survey, and students' motivation was quite possibly higher as they were still in the throes of the semester, taking courses and completing coursework. It was only after the survey was collected that the researchers applied to ENREB to use the anonymous survey data for the purposes of publication. The researchers were granted permission, however, contingent upon approval, they promised that all "future data collection will be started with all informed consents up front." For the second year of the study, the process of ethics approval was not complete until the end of semester due to an unanticipated delay with ENREB. Although surveys were given to team leaders, distribution took place only after the course was complete, and sporadically when team leaders came to drop off peer review forms. It is not known whether team leaders were able to successfully deliver or collect the surveys from their team members; and it is not likely that they were reminding team members about the survey as the course was over. Students were sent two additional email requests, though by that time, the semester was complete. It is quite possible that the timing of the distribution affected the response rate for this year's survey.

Due to the difference in response rate, researchers must consider that self-selection is likely a factor in the kind of findings that were obtained [12]. However, the data will be strengthened by viewing them comparatively with the findings from the first year of the study [6], and with the knowledge that these data will be collected each year to build a longitudinal understanding of students' perceptions of the strengths and weaknesses in the Mechanical Engineering program and of their own aptitudes in regards to the graduate attributes [24].

\subsection{Perceptions of Top Three Program and Student Strengths and Weaknesses (Weighted)}

For the two years the survey was administered, students were asked to rank their top three perceived strengths and weakness and the top three program strengths and weaknesses in regards to the 12 graduate attributes. Scaling the higher responses to a larger value and averaging the total number of responses weighted the data. Figure 1 illustrates these findings. Tables 1 and 2 list the top four program and student strengths and weaknesses respectively, and show how similar the findings from both years were.

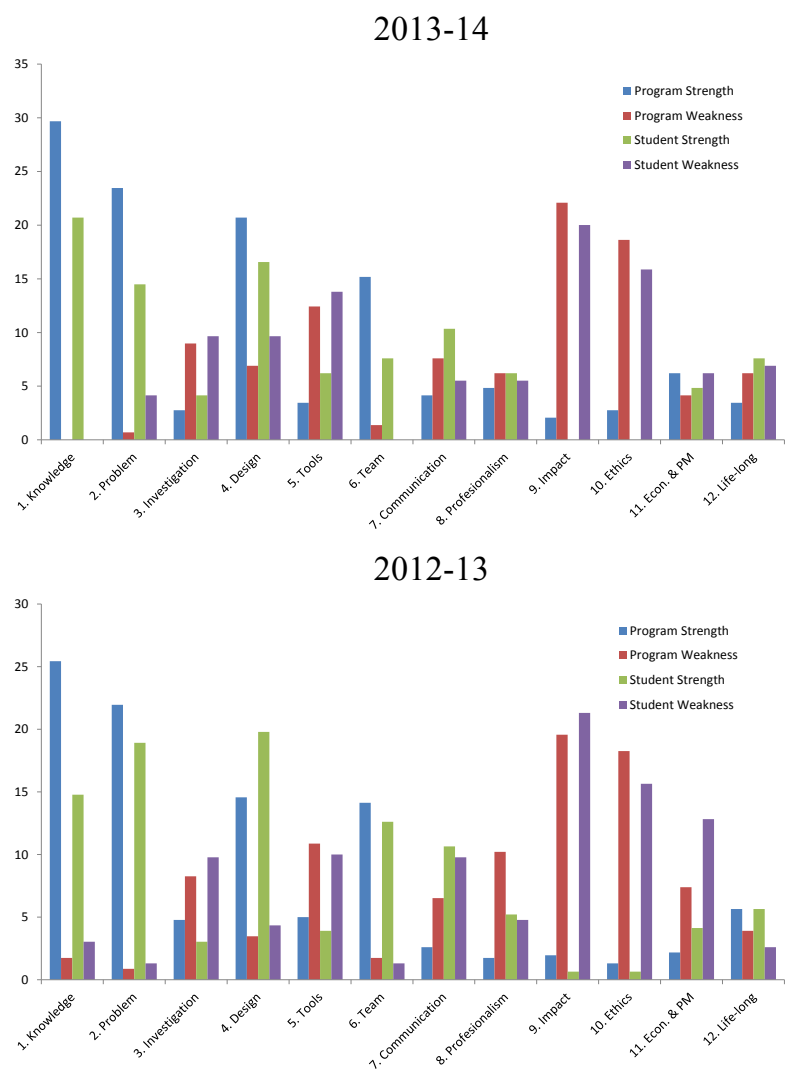

Fig. 1. Weighted percentage of responses for top perceived program strengths and weaknesses, and student strengths and weaknesses.

Table 1. Comparison of weighted percentage of responses of the top four perceived program strengths and weaknesses.

\begin{tabular}{clll}
\multicolumn{1}{c}{ 2013-14 } & \multicolumn{1}{c}{$2012-13$} & \multicolumn{1}{c}{$2013-14$} & \multicolumn{1}{c}{$2012-13$} \\
Program Strength & \multicolumn{1}{c}{ Program Strength } & \multicolumn{1}{c}{ Program Weakness } & \multicolumn{1}{c}{ Program Weakness } \\
Knowledge Base & 1. Knowledge Base & 1. Impact of Eng. & 1. Impact of Eng. \\
. Problem Analysis & 2. Problem Analysis & 2. Ethics \& Equity & 2. Ethics \& Equity \\
Design & 3. Design & 3. Eng. Tools & 3. Eng. Tools \\
Team Work & 4. Team Work & 4. Investigation & 4. Professionalism
\end{tabular}


Table 2. Comparison of weighted percentage of responses of the top four perceived student strengths and weaknesses.

$$
\begin{array}{llll}
\multicolumn{1}{c}{\text { 2013-14 }} & \multicolumn{1}{c}{2012-13} & \multicolumn{1}{c}{2013-14} & \multicolumn{1}{c}{2012-13} \\
\text { Student Strength } & \multicolumn{1}{c}{\text { Student Strength }} & \multicolumn{1}{c}{\text { Student Weakness }} & \multicolumn{1}{c}{\text { Student Weakness }} \\
\text { 1. Knowledge Base } & \text { 1. Design } & \text { 1. Impact } & \text { 1. Impact } \\
\text { 2. Design } & \text { 2. Problem Analysis } & \text { 2. Ethics \& Equity } & \text { 2. Ethics \& Equity } \\
\text { 3. Problem Analysis } & \text { 3. Knowledge Base } & \text { 3. Eng. Tools } & \text { 3. Economics } \\
\text { 4. Communication } & \text { 4. Team Work } & \text { 4. Investigation; } & \text { 4. Eng. Tools } \\
& & \text { Design } &
\end{array}
$$

For weighted percentage of responses for the top four strengths and weaknesses, the perceived program strengths for the first and second year were exactly the same: Knowledge Base for Engineering, Problem Analysis, Design and Individual and Team Work. The first three program weaknesses selected also mirrored each other: Impact of Engineering, Ethics and Equity and Use of Engineering Tools. Investigation and Professionalism were ranked as fourth program weakness for 2013-14 and 2012-13 respectively. The top three student strengths were similar for both years, but in different order: Knowledge Base for Engineering, Design and Problem Analysis for 2013-14, and Design, Problem Analysis and Knowledge Base for Engineering for 201213. The top two ranked student weaknesses were the same: Impact of Engineering on Society and Environment and Ethics and Equity. Use of Engineering Tools was ranked as the third student weakness for 2013-14 and the fourth student weakness for 2012-13. Only one attribute, Design, was ranked as both a student strength and student weakness, and this was only found for 2013-14.

When comparing program and student strength, and program and student weakness, the chosen attributes were generally the same. For 2013-14, program strength reflected student strength, with the exception of Individual and Teamwork (program strength) and Communication Skills (student strength). Program weakness reflected student weakness, with the addition of Design (a student weakness, although as mentioned above, considered a student and program strength as well). Similarly, for 2012-13, program and student strength echoed one another. Program and student weakness were similar, with the exception of Professionalism (program weakness) and Economics and Project Management (student weakness).

\subsection{Perceptions of Top Program and Student Strengths and Weaknesses (Percentage)}

Figure 2 illustrates the percent responses for the top perceived program and student strength and weakness for the two years in which the survey was administered. The graphs on the left are a comparison of program and student strengths and the graphs on the right are a comparison of program and student weaknesses.

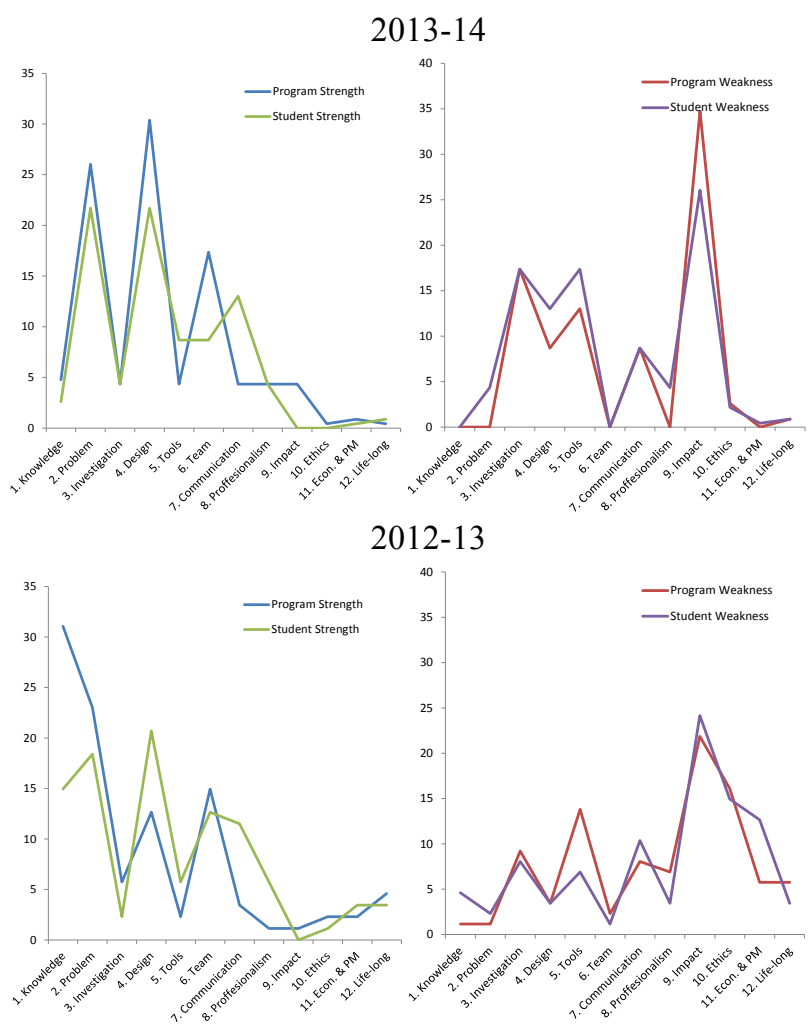

Fig. 2. Percentage of responses for the top perceived program and student strength (left) and program and student weakness (right).

For 2013-14, program and student strengths generally mirrored each other except for Individual and Team Work and Impact of Engineering on Society and the Environment (program higher), and Use of Engineering Tools and Communication Skills (student higher). High strengths were paired with low weaknesses and visaversa, which was a similar finding to 2012-13 [20]. Strengths generally mirrored each other for both years, except for Ethics and Equity, Economics and Project Management, and Lifelong Learning, which showed slight strength for both program and student in 2012-13, and negligible strength in 2013-14. Knowledge Base for Engineering was ranked as a greater program strength than student strength in 2012-13, but was a similar program and student strength in 2013-14. Communication Skills was a greater student strength than program strength in both years. Not one student identified the attribute Impact of Engineering on Society and the Environment in either year as their top strength, and 2013-14 students did not choose Ethics and Equity either.

For 2013-14, program and student weaknesses generally reflected each other, which was similar for the first year of the study, with the exception of Use of Engineering Tools, which was ranked as a larger program weakness than student weakness [20]. 2013-14 students ranked themselves slightly weaker in Knowledge Base for 
Engineering, Problem Analysis, Investigation, Design, Use of Engineering Tools, Economics and Project Management, and Lifelong Learning, and ranked the program weaker in Individual and Team Work, Communication Skills, Professionalism, Impact of Engineering on Society and the Environment, and Ethics and Equity. The trend was that the program was generally ranked weaker than students in the professional skills. This was not found in 2012-13 (Students weaker in Knowledge Base for Engineering and Problem Analysis, Communication Skills, Impact of Engineering on Society and the Environment and Economics and Project Management, and program weaker in Investigation, Use of Engineering Tools, Individual and Team Work, Professionalism and Lifelong Learning [20].) Not one 2013-14 student ranked Knowledge Base for Engineering as a program or student weakness; and not one ranked Individual and Team Work as a student weakness. The program was deemed to be weaker than students in both Individual and Team Work and Professionalism for both years.

Table 3. Comparison of percentage of responses of the top four perceived program strengths and weaknesses. 2013-14 2012-13 2013-14 2012-13 Program Strength Program Strength Program Weakness Program Weaknes 1. Design 1 1. Knowledge Base 1. Impact of Eng. 1. Impact of Eng.

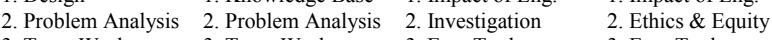

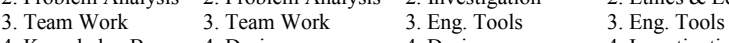
$\begin{array}{lll}\text { 4. Knowledge Base 4. Design } & \text { 4. Design } & \text { 4. Investigation }\end{array}$

Table 4. Comparison of percentage of responses of the top four perceived student strengths and weaknesses 2013-14 2012-13 2013-14 2012-13 Student Strength Student Strength Student Weakness Student Weaknes 1. Problem 1. Design 1 1. Impact of Eng. 1. Impact of Eng. Analysis; Design

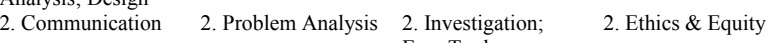

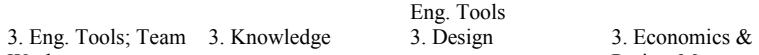
Work $\quad$ Project Managemt 4. Investigation; 4. Team Work 4. Communication 4. Communication Professionalism

When examining the percentage of responses for top four perceived program strengths, students from the first and second year of the study ranked the same four attributes: Design, Problem Analysis, Individual and Team Work and Knowledge Base for Engineering (Table 3). However, 2013-14 ranked Design as the first and Knowledge Base for Engineering as the fourth, and 2012-13 did the opposite. Both years also ranked Impact of Engineering on Society and Environment as the number one program weakness, and Use of Engineering Tools as the third program weakness. Investigation was the second program weakness for 2013-14, and the fourth program weakness for 2012-13.

Problem Analysis and Design were chosen as the top two student strengths for both years, albeit in inverse order (Table 4). Impact of Engineering on Society and the Environment was the number one student weakness for both years.
When comparing program and student strengths, 201213 students chose the same four attributes, although in different order (Table 3 and Table 4). For 2013-14, Design, Problem Analysis and Individual and Team Work were among the top three ranked attributes.

When comparing program and student weaknesses, Impact of Engineering on Society and the Environment is consistently chosen as the top ranked weakness in all categories in both years. This is a trend worth exploring in regards to the program's attribute mapping, course content and curriculum. 2013-14 students perceive Investigation, Use of Engineering Tools and Design respectively for the next ranked program and student weaknesses, (although Design is also ranked as the top program and student strength, as mentioned previously). These data are likely due to the small number of respondents (23) to the comparatively high number of attributes from which to choose (12).

There were other noteworthy findings for the second year of the study. Not one student chose Knowledge Base for Engineering, Problem Analysis, Individual and Team Work, Professionalism, or Economics and Project Management as a top program weakness. All 12 attributes were chosen as top program strength by at least one participant. Impact of Engineering on Society and the Environment and Ethics and Equity were not chosen once as a student strength. Knowledge Base for Engineering and Individual and Team Work was not chosen as a student weakness. And generally, program strengths and student strengths were concentrated in the traditional skills rather than the professional skills, which was a reflection of 2012-13 [20]. However, program and student weaknesses were less concentrated in the professional skills, as opposed to the first year of the study, with the obvious exception of Impact of Engineering on Society and the Environment, which was clearly perceived as both a program and student weakness for both years of the study. (It was also the only attribute not chosen once as a student strength for the first year of the study, which is remarkable considering 76 students responded to the survey during the 2012-13 year [20].)

\subsection{Average Perceived Graduate Competencies}

Figure 3 illustrates the average perceived student competencies for all 12 graduate attributes for each year that the survey was administered. (Note that the scales are different between the two offerings, which is due to the different number of ranking categories students were provided on the two surveys: three categories for the first year and five for the second.) 
2013-14
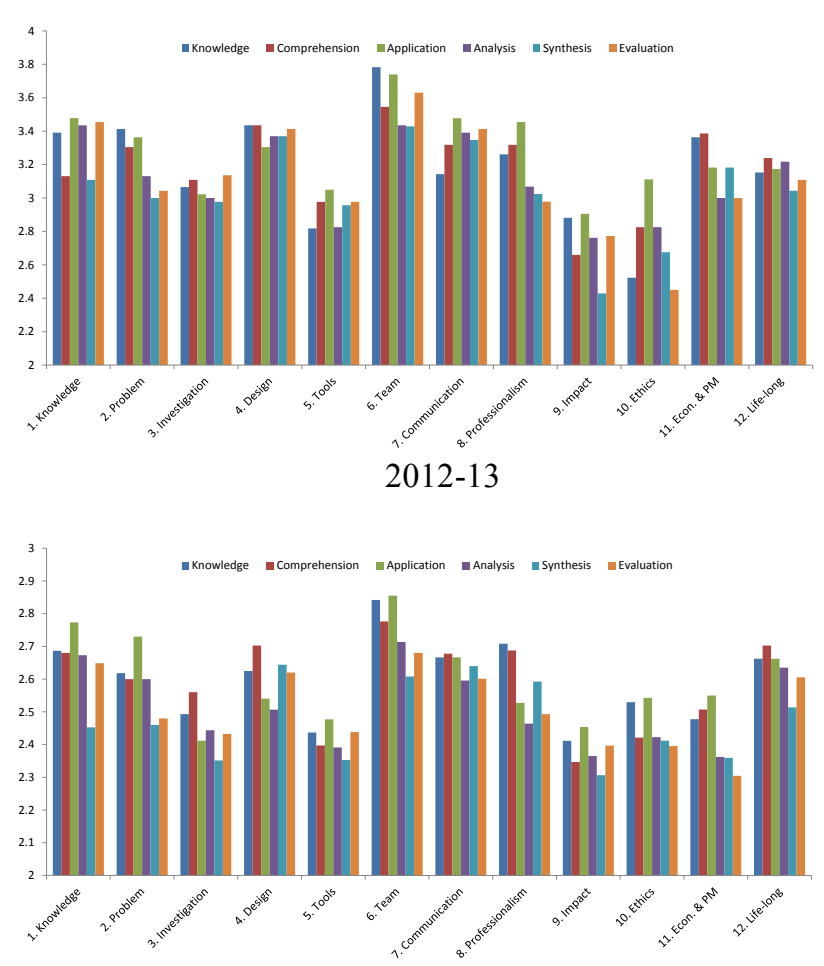

Fig. 3. Average perceived graduate competencies.

For 2013-14, students perceived that they were introduced to the skill/ability within the Mechanical Engineering curriculum, and that their performance was at least "good" for all attributes except for: Use of Engineering Tools, Impact of Engineering on Society and the Environment, and Ethics and Equity. For these, students perceived their performance as "adequate," with Impact of Engineering on Society and the Environment adequate for all six levels in the Cognitive Domain, but Use of Engineering Tools and Ethics and Equity "good" in regards to application. The lowest ranking attribute was Impact of Engineering on Society and the Environment, and the highest rankings were for Individual and Team Work, Design, Communication Skills and Knowledge Base for Engineering. Comparing 2012-13 and 2013-14: Data from both years generally mirrored each other, with the exception of Economics and Project Management, which had a higher ranking in 201314 than in 2012-13. Impact of Engineering on Society and the Environment was ranked lowest for both years. Individual and Team Work was ranked highest and Communication Skills ranked third highest for both years. Knowledge Base for Engineering was ranked second highest for 2012-13 and fourth highest for 2013-14.

\subsection{Close-Up View of Average Perceived Graduate Competencies For Selected Traditional and Professional Attributes}

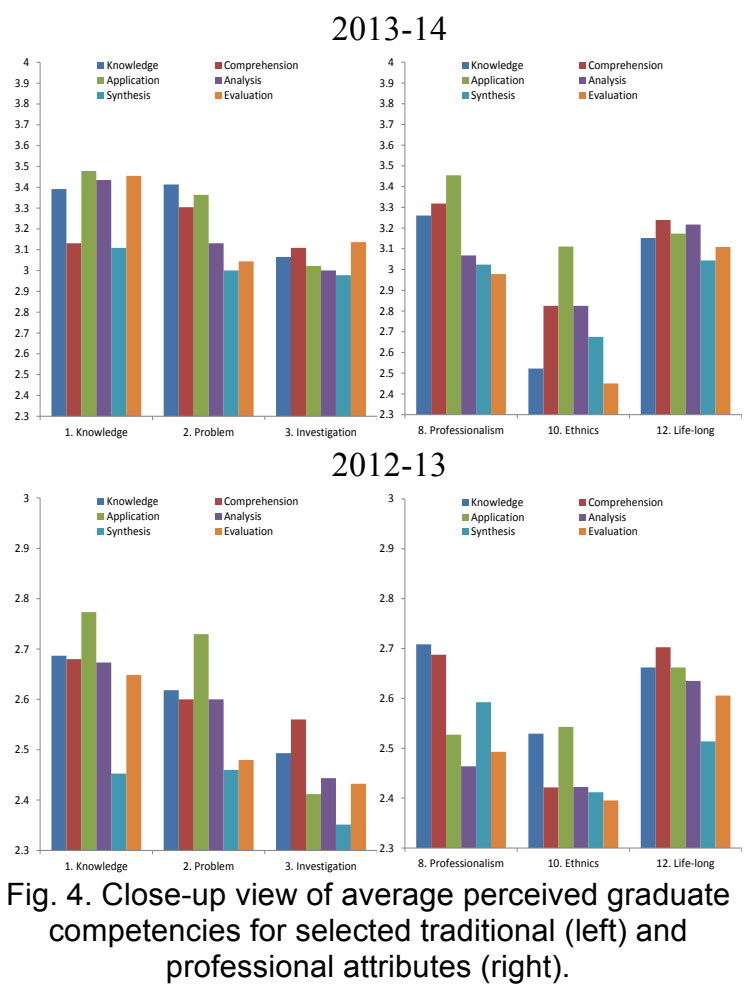

The 12 CEAB attributes are often considered as made up of both traditional skills and professional skills [22]. There is evidence in the research that the traditional skills are assessed more often and more easily than the professional skills [1][3][7][8][10][11][13][22][25][26]. Therefore, to explore whether student perception of strength and weakness reflected a similar trend to support the research, a comparison of the perceived graduate competencies for selected traditional and professional attributes was done. The findings showed that students did not unequivocally find the professional skills weaker than the more traditional skills. For 2013-14, Problem Analysis and Professionalism reflected a drop-off with the higher-level cognitive skills; Knowledge Base for Engineering and Ethics and Equity had inconsistent cognitive rankings; Investigation and Lifelong learning were more equal (Fig. 4). Ethics and Equity was considered "adequate" by students in all but one domain, with five domains falling below the median. The rest of the attributes were all "good" in terms of performance. For 2012-13, only Lifelong Learning had all of its domains on or above the median; the rest had at least one category that fell below the median. Comparing both years, traditional skills mirrored each other, with Knowledge Base for Engineering, Problem Analysis and Investigation ranked higher to lower respectively. For the 
professional attributes, Ethics and Equity was ranked the lowest for both years; but Professionalism was higher than Lifelong Learning for 2013-14 as opposed to 201213 .

\section{DISCUSSION}

Despite the low response rate for this year's survey, the findings examined comparatively with the first year of the study have provided the Mechanical Engineering program and the Engineering Faculty at the University of Manitoba with some important feedback and are unveiling some potential trends worth investigating in the coming years.

For both the weighted percentage of responses for top three perceived program and student strengths and weaknesses and the percentage of responses for the top perceived program and student strengths and weaknesses, Knowledge Base for Engineering, Problem Analysis, Design and Individual and Team Work were consistently chosen as both program and student strengths: three traditional attribute and one professional attribute. Impact of Engineering on Society and the Environment, Ethics and Equity, Use of Engineering Tools and Investigation were fairly steadily ranked as program and student weaknesses: two traditional attributes and two professional attributes.

A faculty attribute assessment study at the University of Manitoba has been ongoing for the past three years, where instructors identify the attributes that they assess in their courses. Data from that research have generally shown that the traditional attributes are assessed more frequently than the professional ones [9][18]. This trend is not reflected in students' perceptions in this study, where the findings show that there is a fairly even distribution of traditional and professional attributes for both program and student strengths and weaknesses. This is somewhat of an unexpected outcome due to the research pertaining to the substantial presence of the traditional skills versus the professional skills in engineering education $[1][3][7][8][10][11][13][22][25][26]$.

\section{LIMITATIONS AND NEXT STEPS}

Caution is advised concerning the methods, particularly with the reliability and validity, or lack thereof, of the survey as an instrument of assessment feedback [23]. There are several studies where the evidence of inconsistency and the wide-range of responses in student exit, faculty and alumni surveys point to the care with which these results should be used [23]. Therefore, it is important to be aware of the potential limitations of the survey as an assessment instrument, which is another reason why it is crucial to have a comprehensive assessment protocol, one that does not rely solely on the results of a single instrument [4][14][24].

Toward this end, the faculty is using a number of other assessment instruments to collect data to inform its continual program improvement commitment. These include a faculty attribute assessment checklist [9][18]; an alumni survey; an Industry survey and forum [5]; and the recent and ongoing development of a set of rubrics designed to assess the 12 graduate attributes at course and program levels [19]. Additionally, as a part of this study, data are also being analyzed from two student focus groups that were recently conducted in concomitance with the student exit survey to explore the findings of the survey from a qualitative perspective. As well, additional data were collected in the exit survey: students were asked to rate their preparedness in regards to each graduate attribute, as well as rate their perception of the importance of each attribute in the engineering profession. These additional data are being gathered to triangulate the findings and strengthen the validity and reliability of the survey.

\section{CONCLUSIONS}

The objective of this study is to examine fourth year Mechanical Engineering students' perceptions of graduate attribute competencies in the Faculty of Engineering at the University of Manitoba. Most of the findings from this year (2013-14) need to be considered with caution due to the low survey response rate. However, it is clear that there are trends emerging when considering the data in partnership with the data from the first year of the study. Deliberating on the findings certainly points to some graduate attributes that need investigating further. Specifically, the attributes Impact of Engineering on Society and the Environment, Ethics and Equity, Use of Engineering Tools and Investigation should be scrutinized within the Mechanical Engineering curriculum, and in conjunction with other stakeholders' perceptions and experiences in light of these findings. In the interim, students' perceptions will continue to be heard and explored at the University of Manitoba's Faculty of Engineering with the commitment to continue to develop and improve its curriculum for the benefit of all stakeholders.

\section{Acknowledgements}

The authors would like to thank the members of our Curriculum Management Committee (CMC) for their support and encouragement, Bernice Ezirim for assisting with data entry, and our fourth year Mechanical Engineering students who participated in this survey. This research study was funded by Jillian Seniuk Cicek's University of Manitoba Graduate Fellowship (UMGF) and Manitoba Graduate Scholarship (MGS). 


\section{References}

[1] Brock E. Barry and Matthew W. Ohland, "Applied ethics in the engineering, health, business, and law professions: A comparison," Journal of Engineering Education, vol. 98, no. 4, pp. 377-388, 2009.

[2] Benjamin, S. Bloom (Ed.), Max D. Engelhart, Edward J. Furst, Walker H. Hill and David R. Krathwohl, Taxonomy of Educational Objectives: The Classification of Educational Goals. Handbook 1: The Cognitive Domain. New York, NY: Longmans, Green \& Co., 1956. 207 pp.

[3] Anne Colby and William M. Sullivan, "Ethics teaching in undergraduate engineering education," Journal of Engineering Education, vol. 97, no. 3, pp. 327-338, 2008.

[4] Amy Driscoll and Swarup Wood, Developing Outcomesbased Assessment for Learning-centered Education: A Faculty Introduction. Sterling, VA: Stylus, 2007, 275 pp. \{ISBN: 978 I 579221959$\}$

[5] Ken Ferens, Jillian Seniuk Cicek, Nariman Sepehri, Witold Kinsner, J.P. Burak, Anne Parker, Dean McNeill, Douglas Ruth, Ian Jeffrey, Norma Godavari, and Sandra Ingram, "Industry Forum III: Towards a common language," in Proc. CEEA Canadian Engineering Education Conf., CEEC14, Sean Maw and Marjan Eggermont (eds.) (Canmore, AB; 811 June 2014), 7 pp., 2014.

[6] Jeffrey A. Gliner, George A. Morgan, and Nancy L. Leech, Research in Applied Settings. New York, NY: Routledge Taylor and Francis Group, 2009 (2nd ed.), 469 pp. \{ISBN: 978-0-8058-6434-2\}

[7] James H. Hanson and Julia M. Williams, "Using writing assignments to improve self-assessment and communication skills in an engineering statics course," Journal of Engineering Education, vol. 97, no. 4, pp. 515-529, 2008.

[8] Matthew A. Holsapple, Donald D. Carpenter, Janel A. Sutkus, Cynthia J. Finelli, and Trevor S. Harding, "Framing faculty and student discrepancies in engineering ethics delivery," Journal of Engineering Education, vol. 101, no. 2, pp. 169-186, 2012.

[9] Sandra Ingram, Jillian Seniuk Cicek and Nariman Sepehri, "The Attribute assessment process at the University of Manitoba," in Proc. CEAA Canadian Engineering Conf., CEEC12, (Winnipeg, MB; 17-21 June 2012), 7 pp., 2012.

[10] Scott Jiusto and David DiBiasio, D., "Experiential learning environments: Do they prepare our students to be selfdirected, life-long learners?," Journal of Engineering Education, vol. 95, no. 3, pp. 195-204, 2006.

[11] David H. Jonassen, Demei Shen, Rose M. Marra, YoungHoan Cho, Jenny L. Lo and Vinod Lohani, "Engaging and supporting problem solving in engineering ethics," Journal of Engineering Education, vol. 98, no. 3, pp. 235-254, 2009.

[12] Paul J. Lavrakas, Encyclopedia of Survey Research
Methods. SAGE Research Methods, 2008. \{ISBN: 9781412963947\} Available as of April 13, 2014 from http://srmo.sagepub.com/view/encyclopedia-of-surveyresearch-methods/n526.xml

[13] Michael C. Loui, “Assessment of an engineering ethics video: Incident at Morales," Journal of Engineering Education, vol. 95, no. 1, pp. 85-91, 2006.

[14] Jack McGourty, Catherine Sebastian, and William Swart, "Developing a comprehensive assessment program for engineering education," Journal of Engineering Education, vol. 87 , no. 4, pp. 355-361, 1998.

[15] Charles K. Parsons, Evan Caylor, and Harold S. Simmons, "Cooperative education work assignments: The role of organizational and individual factors in enhancing ABET competencies and co-op workplace well-being," Journal of Engineering Education, vol. 94, no. 3, pp. 309-317, 2005.

[16] Gloria M. Rogers, "EC2000 and measurement: How much precision is enough?," Journal of Engineering Education, vol. 89, no. 2, pp. 161-166, 2000.

[17] Katherine Scales, Christi Owen, Subodh Shiohare, and Michael Leonard, "Preparing for program accreditation review under ABET engineering criteria 2000: Choosing outcome indicators," Journal of Engineering Education, vol. 87, no. 3, pp. 207-210, 1998.

[18] Jillian Seniuk Cicek, Sandra Ingram, and Nariman Sepehri, "The attribute assessment process at the University of Manitoba: Year two," in Proc. CEEA Canadian Engineering Education Conf., CEEC13, Michel Perrier and Sylvie Dore (eds.) (Montreal, QC; 17-20 June 2013), 7 pp., 2013.

[19] Jillian Seniuk Cicek, Sandra Ingram, Nariman Sepehri, J.P. Burak, Paul Labossiere, Danny Mann, Douglas Ruth, Anne Parker, Ken Ferens, Norma Godarvi, Jan Oleszkiewicz, and Aidan Topping. "Rubrics as a Vehicle to Define the Twelve CEAB Graduate Attributes, Determine Graduate Competencies, and Develop a Common Language for Engineering Stakeholders," in Proc. CEEA Canadian Engineering Education Conf., CEEC14, Sean Maw and Marjan Eggermont (eds.) (Canmore, AB; 8-11 June 2014), 6 pp., 2014.

[20] Jillian Seniuk Cicek, Paul Labossiere, and Danny Mann, "Surveying fourth year engineering student perceptions of graduate attribute competencies," in Proc. CEEA Canadian Engineering Education Conf., CEEC13, Michel Perrier and Sylvie Dore (eds.) (Montreal, QC; 17-20 June 2013), 7 pp., 2013.

[21] Joseph Shaeiwitz, "Outcomes assessment in engineering education," Journal of Engineering Education, vol. 85, no. 3, pp. 239-246, 1996.

[22] Larry J. Shuman, Mary Besterfield-Sacre and Jack McGourty, "The ABET "professional skills" - can they be taught? Can they be assessed?," Journal of Engineering Education, vol. 94, no. 1, pp. 41-55, 2005. 
[23] Neelam Soundarajan, "Preparing for accreditation under EC 2000: An experience report," Journal of Engineering Education, vol. 91, no. 1, pp. 117-123, 2002.

[24] Joni E. Spurlin, Sarah A. Rajala, and Jerome P. Lavelle, Designing Better Engineering Education Through Assessment. Sterling, VA: Stylus, 2008, 360 pp. [ISBN: 978I-57922-212-3\}

[25] Bugrahan Yalvac, H. David Smith, John B. Troy, and Penny Hirsch, "Promoting advanced writing skills in an upper level engineering class," Journal of Engineering Education, vol. 96, no. 2, pp. 117-128, 2007.

[26] Carman R. Zafft, Stephanie G. Adams, and Gina S. Matkin, "Measuring leadership in self-managed teams using the competing values framework," Journal of Engineering Education, vol. 98, no. 3, pp. 273-282, 2009.

\section{APPENDIX A: RATING SCALE FOR THE STUDENT EXIT SURVEY}

The " 0 " indicates that you were not introduced to the skill/ability within the Mechanical Engineering curriculum.

The " 1 " indicates that you were introduced to the skill/ability within the Mechanical Engineering curriculum, but that your performance is poor.

The " 2 " indicates that you were introduced to the skill/ability within the Mechanical Engineering curriculum, and that your performance is adequate.

The " 3 " indicates that you were introduced to the skill/ability within the Mechanical Engineering curriculum, and that your performance is good.

The "4" indicates that you were introduced to the skill/ability within the Mechanical Engineering curriculum, and that your performance is excellent. 